\title{
Acoustic versus assoclative models of short-term memory coding
}

JOHN F. MARSHALL, RICHARD O. ROUSE, JR., and ROGER M. TARPY, Williams College, Williamstown, Mass. 01267

A 230-item word list was used to study methods of coding. The list consisted of 35 pivot words, one each of their high associates (HA), medium associates (MA), synonyms (S), rhymes $(R)$, plus 20 buffer words. The data indicated that short-term memory coding complied to an associative rather than an acoustical model. The study offers a possible explanation of why different experiments have found acoustical coding predominant in short-term memory.

McGeoch \& McDonald (1931) were the first to examine the methods of encoding over short intervals of time. They found that the degree of forgetting was related to the degree of associative similarity between the interpolated task and the original stimulus. In contrast, recent studies by Conrad (1962, 1964) and Wickelgren (1966) have hypothesized an acoustic coding model. Conrad (1962) and Wickelgren (1966) have found that the interference in memory comes mostly from different consonants of the same acoustical set. Conrad suggested that when the $S$ perceives a particular consonant, a memory trace is formed. As this trace decays, acousticallysimilar errors result due to the presence of neurological noise within the recall system of the $S$. Conrad (1964), in extending his research to include visually presented material, found that the $S$, upon seeing a consonant, covertly rehearses it, thus encoding the consonant by its acoustical dimension. Wickelgren (1965) has further shown that when such rehearsal is prevented, $S$ 's performance is impaired because the chance of acoustical coding is reduced.

An associative model for short-term memory coding has been suggested recently by several studies. Kolers (1966) observed that bilingual $S s$ are able to recall a word equally well when its other-language translation is substituted for some of its repetitions, despite the fact that the item and its translation may be visually and phonetically different. From this result it was argued that what is stored in short-term memory is not a word in a particular language, but a concept or meaning. Experiments by Rouse \& Verinis (1962) and Schwartz \& Rouse (1961) on visual perception further suggested that each stimulus word activates a "cluster" or "halo" of associations within the memory trace.

The two models of short-term memory were compared within a single experiment by Conrad, Freeman, \& Hull (1965). They concluded that, for short-term storage of six-consonant sequences, encoding depends much more upon acoustical factors than upon their frequency of usage in language. The authors did, however, suggest that when the letter-sequence is of a higher order (that is, when the letter sequence occurs more frequently in the language) then associational factors become more significant.

The purpose of the present experiment was to test the relative importance of the acoustical and associative methods of coding in the short-term memory of higher order letter-sequences (common words). It was anticipated that associational coding would be predominant over acoustical coding because of the nature of the stimuli.

\section{SUBJECTS}

Forty-two members of the introductory psychology class at Williams College were used as $S s$ for this experiment. Three $S s$ were discarded from the analysis for failing to follow instructions.

\section{MATERIALS}

A tape-recording was made, consisting of a list of 230 words common in daily usage. The median rate of occurrence was 50-100 times per million (Thorndike \& Lorge, 1944). The first 55 words on the list were 35 pivot words randomly interspersed with 20 buffer words. The rest of the list consisted of one repetition of each pivot word, its high associate (HA), medium associate (MA), synonym (S), and rhyme (R), randomly ordered. The pivot words, their high associates, and their medium associates were all taken from Palermo \& Jenkins (1964). The mean rate of association for the HAs was 169.7, and for the MAs, 63.6 (Palermo \& Jenkins, 1964). The synonyms were words with approximately identical meanings as the pivot words, but not associates.

\section{PROCEDURE}

The $S s$ were instructed to listen to the list. They were told that some of the words were repeated while others were not. The words were read at the rate of one every $3 \mathrm{sec}$. During that time, $S s$ were to indicate on their answer sheets whether or not the word had appeared previously in the list. Also, the $S$ was to indicate, by the use of the numbers $1,2,3,4$, or 5 , the relative confidence of his judgment. The number 1 indicated extreme lack of confidence, while 5 indicated extreme confidence. An example was given to insure that all $S s$ understood the procedure.

\section{RESULTS}

Only the errors in which the $S$ thought he had heard the word previously in the list (intrusion errors) were considered, since those errors in which the $S$ failed to recognize pivot words (errors of omission) were not considered relevant to the study. The mean frequencies of error, per $S$, were 9.41 (HA), 7.77 (MA), 5.95 (S), and 4.47 (R). An analysis indicated that intergroup differences were highly significant $[F(3,152)=$ $7.328 ; \mathrm{p}<.001]$. Accordingly, t-tests were used to examine differences between each pair of groups. From this analysis it was found that the HA intruded significantly more frequently than did the MA, $S$, or $R$ groups. The MA intruded significantly more than did either $S$ or $R$. Finally, $S$ intruded significantly more frequently than did $R$. In all cases, the tests were significant at the .01 level.

The mean level of confidence for each type of error was then analyzed. No significant effect was found $[F(3,148)=$ $2.254 ; \mathrm{p}>.05]$. The confidence level scores were then changed to deviation scores to compensate for individual differences in the use of the confidence rating score, but still no effect was found. The mean confidence level for all correct responses (3.91) was tested against the mean confidence level for all incorrect responses (3.41) regardless of their type. This difference was found to be highly significant $[\mathrm{t}(38)=4.05$; $\mathrm{p}<.001]$.

The frequency of errors, regardless of their type, was found to increase over the course of testing. Analysis confirmed [ $\mathrm{t}(8)$ $=4.95 ; p<.01]$ that the total number of errors on the first half of the word list (485) where intrusion errors could have occurred was significantly less than the total number of errors on the second half $(585)$.

\section{DISCUSSION}

The results of this experiment support the conclusion that for short-term recall of words, associational coding plays a significantly greater role than does acoustical coding. This conclusion is based on the finding that HA errors were most frequent. MA, S, and $R$ were respectively less often confused with the pivot word. As suggested by Conrad et al (1965), it seems that the prevalence of the associative vs acoustical 
coding method within $S s$ depends, at least in part, upon the material to be stored. When the verbal sequences to be encoded are met in everyday usage, such as common words, then the associational coding dimension becomes increasingly important. The present findings, when considered along with the Conrad et al (1965) study, suggest that an item to be stored is coded by arousing a cluster of both associativelysimilar and acoustically-similar items. The relative strengths of these two types of coding depend on the nature of the recall item.

The use of the confidence level as an indication of error differences between high associates, medium associates, synonyms, and thymes was not successful. The authors predicted a lower level of confidence for the HA, which had the most errors, and the highest level of confidence for $R$. The failure of this measure to correspond with the error analysis cannot be explained by the individual differences in the use of the confidence level, since deviation scores produced similar results. When the mean confidence level for all correct responses was tested against the mean confidence level for all incorrect responses, however, a significant difference was found, indicating that when a $S$ was correct, he was generally more confident in his response, regardless of the type of coding used.

Finally, evidence indicated overall recall decreased during the course of testing. The farther along on the list an error occurred, the more time there had been for the trace to decay since the presentation of the pivot word. Thus, errors on any given item were dependent not only on its group (HA, MA, S, or $\mathrm{R}$ ), but also on its position in the list. Since the $S s^{\prime}$ covert rehearsal strategies were not controlled, it is not possible to determine whether this position effect was a function of interitem interferences or of autonomous decay.

\section{REFERENCES}

CONRAD, R. An association between memory errors and errors due to acoustic masking speech. Nature, 1962, 193, 1314-1315.

CONRAD, R. Acoustic confusions in immediate memory. British Journal of Psychology, 1964, 55, 75-84.

CONRAD, R., FREEMAN, P. R., \& HULL, A. J. Acoustic factors versus language factors in short-term memory. Psychonomic Science, 1965, 3, 57-58.

KOLERS, A. Reading and talking bilingually. American Journal of Psychology, 1966, 79, 357-376.

McGEOCH, J. A., \& McDONALD, W. T. Meaningful relation and RI. American Journal of Psychology, 1931, 43, 579-588.

PALERMO, D. S., \& JENKINS, J. J. Word association norms. Minneapolis: University of Minnesota Press, 1964.

ROUSE, R. O., \& VERINIS, J. S. The effect of associative connections on the recognition of flashed words. Journal of Verbal Learning \& Verbal Behavior, 1962, 1, 300-303.

SCHWARTZ, F., \& ROUSE, R. 0 . The activation and recovery of associations. Psychological Issues, 1961, 3, 1-141.

THORNDIKE, E. L., \& LORGE, I. The teacher's handbook of 30,000 words. New York: Teachers College, Columbia University, 1944.

WICKELGREN, W. A. Acoustic similarity and intrusion errors in short-term memory. Journal of Experimental Psychology, 1965, 70, 102-108.

WICKELGREN, W. A. Short-term recognition memory for single letters and phonemic similarity in RI. Quarterly Joumal of Experimental Psychology, 1966, 18, 55-62.

(Contimued from page 53)

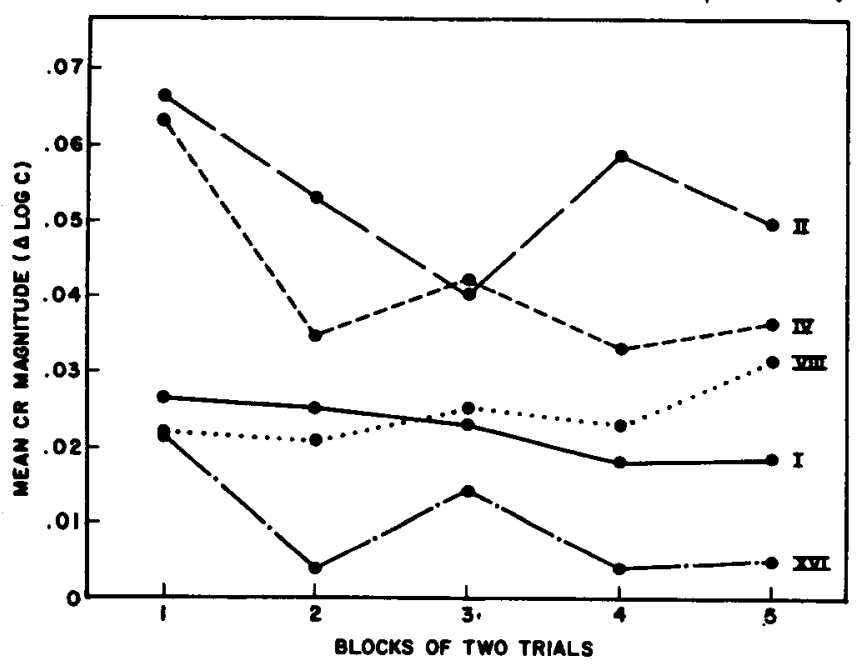

Fig. 2. Mean GSR magnitude during extinction for the five conditioning groups, in five blocks of two trials.

\section{DISCUSSION}

Except for the group that received one postpeak paired-conditioning trial, the results supported the hypothesis that resistance to extinction would vary inversely with the number of postpeak acquisition trials. The unexpectedly lower resistance to extinction in Group 1 may have been due to one of two possible factors. First, it is likely that a single smaller response does not provide a reliable index of the location of the peak CR. It may be that the unreliability of using only a single trial to identify the peak $C R$ resulted in premature termination of acquisition in Group 1. Group 1 received, on the average, only four to five paired trials altogether. ${ }^{2}$ A second possibility is that omission of the UCS following only a single trial of reduced response may have resulted in instrumental reinforcement of reduced responding (i.e., via avoidance), producing even more reduced responding on subsequent trials. Parsimony would suggest that the former of these post hoc explanations be considered the more viable.

The inverse relationship between resistance to extinction and number of postpeak acquisition trials implies that inhibition of delay generated during acquisition trials adds to the response-attenuation produced by extinction. Since inhibition of delay and extinctive inhibition are also both vulnerable to disinhibition by means of a novel stimulus, they may reflect the same underlying neurological process.

The present results suggest that each $S$ in classical GSR conditioning be given a number of paired trials uniquely appropriate to his own developing excitatory and inhibitory tendencies, if the aim of the experiment is to achieve a high level of resistance to extinction. The conventional procedure of giving a fixed number of trials to all Ss, even if this number is small, obviously entails the possibility of giving some Ss too many paired trials and giving others too few.

It should also be noted that Group 16 showed no more resistance to extinction than did Group $C$, even though the groups differed substantially after the first few acquisition trials. Group 16 received some 19-20 paired trials, an amount that does not differ greatly from what is given in many conventional GSR conditioning studies. Thus, an individually tailored number of paired trials has been shown in this study to result in increased resistance to extinction, while a large but conventional number of trials has been shown to result in extinction performance that does not even support the conclusion that conditioning occurred.

\section{NOTES}

1. Based upon the first author's M.A. thesis, done at Ohio University under the second author's supervision and supported by USPHS Grant MH-12262-04.

2. The possibility that the conditioning groups differed even prior to conditioning as a result of sampling was also examined but found to be entirely negligible. 